\title{
Neuer Insulin-Pen verbindet Funktionalität, Individualität und Ästhetik
}

\author{
Um insulinpflichtigen Diabetikern die Therapie zu erleichtern, \\ ist neben modernen Insulinen auch eine optimale technische \\ Unterstützung erforderlich. Mit dem neuen Insulin-Pen halten \\ nun auch ästhetische Aspekte in die Insulintherapie Einzug.
}

\begin{abstract}
Verbesserungsbedarf beim Management des Diabetes mellitus besteht vor allem in Bezug auf die verordneten Insulinregime, die sich für viele Patienten nur schwer mit Bedürfnissen und Alltagsbedingungen in Einklang bringen lassen, sagte Prof. Petra-Maria Schumm-Draeger aus München. Dass die Insulintherapie noch optimierungsbedürftig ist, bestätigen die Ergebnisse der weltweiten Umfrage GAPP $^{\mathrm{TM}}$ (Global Attitudes of Patients and Physicians in Insulin Therapy) bei 1530 Typ-1- und Typ-2-Diabetikern und 1250 Ärzten. Demnach lassen 35\% der Patienten manchmal eine Insulindosis aus. 77\% der Ärzte nehmen an, dass die Patienten jeden Monat bis zu 6 Injektio-
\end{abstract}

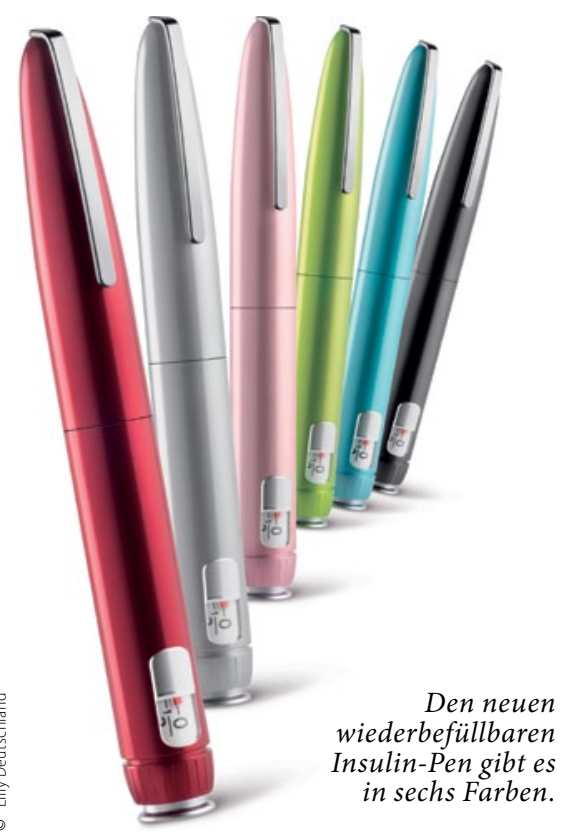

nen versäumen. Eine optimale Blutzuckerkontrolle erfordere moderne Präparate mit gutem Sicherheitsprofil, die sich wie Insulinlispro (Humalog ${ }^{*}$ mit den $\mathrm{Pa}$ tientenbedürfnissen in Einklang bringen lassen und die Flexibilität der Therapie erhöhen, so Schumm-Draeger.

Injektionshilfen tragen dazu bei, den Komfort zu verbessern und die Sicherheit zu erhöhen. Mit der Einführung des neuen Insulin-Pen (HumaPen Savvio) wurden nun auch ästhetische Aspekte berücksichtigt. Der einfach zu bedienende, wiederauffüllbare Pen hat ein modernes Design. Die Farbvielfalt biete Individualität und helfe dem Patienten dabei, verschiedene Insuline zu unterscheiden, so

Emine Belek, Diabetesberaterin in München. Er ist leichter und kürzer als andere Pens und ähnelt einem Kugelschreiber. So ist eine unauffällige, diskrete Nutzung möglich.

Abdol A. Ameri

\section{Anti-VEGF-Therapie wird am besten bedarfsorientiert gestaltet}

\section{Werden Patienten mit feuchter altersbedingter Makuladegenera- tion (AMD) nur bei Bedarf mit einem vaskulären endothelialen Wachstumsfaktor-(VEGF-)Hemmer wie Ranibizumab behandelt, minimiert dies ihr Endophthalmitisrisiko.}

Ein fixes Schema für Patienten, die wegen feuchter AMD mit einem VEGF-Inhibitor behandelt werden? Nach Einschätzung von Prof. Focke Ziemssen von der Universitäts-Augenklinik Tübingen ist dies nicht sinnvoll. Denn die Patienten sprechen individuell sehr unterschiedlich an.

Als Beispiel zog Ziemssen Daten von AMD-Patienten heran, die im Rahmen der HARBOR-Studie Ranibizumab 0,5 mg (Lucentis ${ }^{\circledast}$ ) erhalten hatten - zunächst drei monatliche Injektionen, gefolgt von einer bedarfsorientierten Therapie (pro re nata, PRN). Nach zwei Jahren hatte sich der Visus der Patienten im
Vergleich zum Studienbeginn um durchschnittlich 7,9 Buchstaben verbessert (Busbee BG. Retina Subspecialty Day zum Kongress der „American Academy of Ophthalmology” (AAO), Chicago, 9. 13.11.2012, mdl. Vortrag). Die mittlere Zahl der Injektionen betrug 7,7 im ersten und 5,6 im zweiten Jahr - insgesamt also 13,3. „Etwa 30\% der Patienten benötigten in zwei Jahren aber weniger als zehn Injektionen“, gab Ziemssen zu bedenken. Hätten sie nach einem fixen Schema mehr Injektionen erhalten, wären sie übertherapiert und einem unnötig hohen Endophthalmitisrisiko ausgesetzt worden. Die deutschen Fachgesellschaften empfehlen unabhängig vom eingesetzten VEGF-Inhibitor eine bedarfsorientierte Therapie. Zwischen den Injektionen sollte die Krankheitsaktivität monatlich mittels Spektraldomänen Optische Kohärenztomografie (SD-OCT) kontrolliert werden.

Nach Daten der Kölner UniversitätsAugenklinik ist die Adhärenz der AMDPatienten bei einer Ranibizumab-Therapie hoch [1]. Mehr als 90\% würden sich noch einmal für die Behandlung entscheiden. Bei Patienten mit diabetischem Makulaödem belegen 3-Jahres-Daten der RESTORE-Studie, dass sich die initial mit Ranibizumab erzielten Visusverbesserungen mit einer PRN-Therapie langfristig halten lassen [2].

Dr. Matthias Herrmann

1. Droege KM et al. Graefes Arch Clin Exp Ophthalmol 2012; Epub ahead of print

2. Mitchell P et al. Kongress der AAO 2012, Poster P0532

Pressegespräch bei der Tagung der Augenärztlichen Akademie Deutschlands (AAO) 27.2.2013 in Düsseldorf, Veranstalter: Novartis 